\title{
Estabilidad temporal de las infracomunidades de parásitos en la borrachilla Scartichthys viridis (Valenciennes, 1836) (Pisces : Blenniidae) en la costa central de Chile
}

\author{
Temporal stability in parasite infracommunities of the blenny Scartichthys viridis \\ (Valenciennes, 1836) (Pisces: Blenniidae) on the central coast of Chile
}

\section{FREDDY DÍAZ \& MARIO GEORGE-NASCIMENTO}

Facultad de Ciencias, Universidad Católica de la Santísima Concepción, Casilla 297, Concepción, Chile; e-mail: mgeorgen@ucsc.cl

\begin{abstract}
RESUMEN
Se comparan las infracomunidades de parásitos de la borrachilla Scartichthys viridis (Pisces: Blenniidae) de tres muestras tomadas en un lapso de 17 meses, entre 1999 y 2001, desde pozas intermareales vecinas a Las Cruces ( $33^{\circ} 27^{\prime}$ $\left.\mathrm{S}, 71^{\circ} 37^{\prime} \mathrm{O}\right)$, Chile central. El objetivo es averiguar si la riqueza, diversidad, abundancia y composición de las infracomunidades de parásitos son persistentes o no en el tiempo ontogenético y cronológico. Se recolectaron 10 taxa parasitarios en cerca del $89 \%$ de los 63 huéspedes examinados, de los cuales ocho eran metazoarios. La búsqueda de protozoos se realizó sólo en los últimos 2 años. El $75 \%$ de los ocho taxa de metazoos ocurrieron en los tres años, uno no estuvo presente en un año, y otro fue encontrado solamente en un año. La mayoría de los huéspedes examinados albergaba protozoos parásitos. La prevalencia de los protozoos Cryptobia sp. fue alta para ambos años, y menor en Trichodina sp. La longitud total de los huéspedes era un predictor relevante de las variaciones en la riqueza, abundancia, diversidad y composición infracomunitaria en el conjunto de los tres años. En cambio, el año de muestreo no lo fue, en particular al comparar entre huéspedes de similar longitud total. Estos resultados son similares a los encontrados en infracomunidades de parásitos de otras especies de peces en Chile, en que hay baja variabilidad en las propiedades agregadas de las infracomunidades en el tiempo cronológico. Se discute acerca de la extensión relativa del tiempo ontogenético en contraste al tiempo cronológico al ser probablemente ambos de importancia en las características de las infracomunidades, y se propone que se explore la medida en la cual estos resultados constituyen un patrón.
\end{abstract}

Palabras clave: estabilidad temporal, infracomunidades de parásitos, Scartichthys viridis, Chile.

\begin{abstract}
Parasite infracommunities of the blenny Scartichthys viridis (Pisces: Blenniidae) are compared among three samples taken within a 17-month period during three consecutive years (1999-2001), from intertidal pools at Las Cruces ( $33^{\circ} 27^{\prime}$ S, $\left.71^{\circ} 37^{\prime} \mathrm{W}\right)$, central Chile. Our goal is to assess if the richness, diversity, abundance and composition of parasite infracommunities are persistent or not through chronological time, as well as throughout the ontogeny of this herbivorous intertidal blenny. Ten parasitic taxa were found in ca. $89 \%$ of all 63 host blennies examined, eight of which were metazoans. Protozoans were looked for in the last 2 years of the study only. Seventy five percent of all eight metazoan taxa occurred in all three years, one was absent in one year, and another was present in one only. Most of the blennies harbored protozoans. Prevalence of protozoan Cryptobia sp. was high in both years and lower in Trichodina sp. In the overall sample, total host body length was a relevant predictor of the variations in composition, richness, total abundance and diversity of parasite infracommunities. In contrast, the year of sampling was not, especially when infracommunities compared came from hosts of similar body size. These results agree with those of parasite infracommunities found in other fish host species in Chile, in that there is low compositional and aggregated variability along chronological time. The relative extension of ontogenetic versus chronological time is a subject to be explored further with respect to the amount of differentiation in these assemblages.
\end{abstract}

Key words: temporal stability, parasite infracommunities, Scartichthys viridis, Chile.

\section{INTRODUCCIÓN}

En el estudio de la ecología de las comunidades biológicas se pueden distinguir niveles, todos igualmente legítimos, que pueden ser definidos, por ejemplo, en una jerarquía de hábitats (ver
Begon et al. 1990). Precisamente, una aproximación al estudio de las comunidades de parásitos consiste en considerar a cada individuo huésped como portador de una réplica de la comunidad componente de parásitos que habita en esa población o especie. A tales réplicas se las denomina 
infracomunidades y en ellas se puede indagar, por ejemplo, si las características de la distribución y abundancia de los parásitos en los individuos huéspedes son propias de unidades de estudio en las cuales es probable detectar procesos de interacción entre ellas (George-Nascimento \& Iriarte 1989). Otra aproximación consiste en considerar la muestra de la población o especie huésped como unidad de estudio, para compararla con muestras de otras poblaciones o especies huéspedes, en sus descriptores comunitarioparasitológicos (e.g., Kennedy et al. 1986).

Para evaluar si las comunidades son persistentes, se dice que se debe considerar el número de especies, la conectancia o número de conexiones entre especies como fracción del total posible, y la magnitud de las interacciones entre especies (ver Putman 1994). Las infracomunidades parasitarias, sin embargo, poseen pocas especies y las interacciones entre y dentro de niveles tróficos serían casi inexistentes (ver Kuris \& Lafferty 1994, Sousa 1994 para excepciones). Altos valores de conectancia o de magnitud de las interacciones conferirían inestabilidad. Esto quiere decir que ensambles especiosos debieran tener baja conectancia y/o interacción para poder persistir (ver Jaksic 2001). La persistencia de las infracomunidades de endoparasitos transmitidos tróficamente ha sido entendida como el reflejo de la estabilidad de las relaciones tróficas y uso del hábitat de los huéspedes (Garcías et al. 2001). Algunos pocos estudios en la variabilidad temporal de las infracomunidades de parásitos en peces marinos han evaluado la similitud entre años consecutivos en composición, diversidad y otros descriptores infracomunitarios (Kennedy 1993, Thoney 1993, Balboa \& George-Nascimento 1998), excepto Garcías et al. (2001) que lo hicieron en una misma localidad y huésped, con 15 años de diferencia. La variabilidad temporal de las infracomunidades de parásitos ha sido recientemente examinada en ensambles de nemátodos que habitan en el tracto digestivo de ovejas, donde se observaron variaciones no significativas en la diversidad, equitabilidad y riqueza de especies entre años, aunque hubo cambios menores en composición. Estos resultados indican una gran estabilidad de las infracomunidades, tanto en el tiempo cronológico (estaciones del año) como a través de diversas características del huésped, como la edad o el sexo (Gruner et al. 1998).

La especie de huésped de la comunidad de parásitos que es objeto de este estudio es la borrachilla Scartichthys viridis (Valenciennes, 1836) (Pisces: Blenniidae). Como ella no experimenta cambios ontogenéticos sustantivos en su dieta y en el uso del hábitat, cabría esperar que los cambios ontogenéticos en la composición de las

\section{TABLA 1}

Prevalencia (\%) y abundancia (DE, desviación estándar) de 10 taxa de parásitos encontrados en 63 ejemplares de borrachilla Scartichthys viridis muestreadas entre 1999 y 2001 en Las Cruces, Chile; $(-)=$ datos no disponibles

Prevalence (\%) and abundance (standard deviation, DE) of 10 parasite taxa found in 63 blenny Scartichthys viridis individuals sampled between 1999 and 2001 at Las Cruces, Chile; (-) = data not available

\begin{tabular}{|c|c|c|c|c|c|c|}
\hline \multirow[b]{2}{*}{ Parásitos } & \multicolumn{2}{|c|}{$\begin{array}{l}\text { Año } 1999 \\
(\mathrm{n}=30)\end{array}$} & \multicolumn{2}{|c|}{$\begin{array}{l}\text { Año } 2000 \\
(\mathrm{n}=18)\end{array}$} & \multicolumn{2}{|c|}{$\begin{array}{l}\text { Año } 2001 \\
(\mathrm{n}=15)\end{array}$} \\
\hline & Prevalencia & Abundancia & Prevalencia & Abundancia & Prevalencia & Abundancia \\
\hline Hirudinea & & & & & & \\
\hline $\begin{array}{l}\text { Oceanobdella sp. } \\
\text { Copepoda }\end{array}$ & 76,7 & $2,8(3,2)$ & 72,2 & $2,4(2,6)$ & 66,7 & $1,2(1,2)$ \\
\hline Lepeophtheirus sp. & 10,0 & $0,1(0,3)$ & 44,4 & $0,7(0,9)$ & 33,4 & $0,3(0,5)$ \\
\hline $\begin{array}{l}\text { Acantochondria sp. } \\
\text { Monogenea }\end{array}$ & 0 & 0 & 0 & 0 & 6,7 & $0,1(0,5)$ \\
\hline Neobenedenia melleni & 10,0 & $0,1(0,4)$ & 5,6 & $<0,1(0,2)$ & 40,0 & $1,1(2,1)$ \\
\hline $\begin{array}{l}\text { Paramicrocotyle moyanoi } \\
\text { Nematoda }\end{array}$ & 16,7 & $0,3(0,9)$ & 61,1 & $1,7(2,4)$ & 53,3 & $2,7(2,9)$ \\
\hline Spirurida & 13,3 & $0,2(0,6)$ & 11,1 & $0,1(0,3)$ & 6,7 & $0,07(0,3)$ \\
\hline Digenea & & & & & & \\
\hline Sp. 1 & 16,7 & $0,3(0,6)$ & 33,3 & $1,1(1,9)$ & 33,4 & $0,7(1,9)$ \\
\hline $\begin{array}{l}\text { Lepidauchen } \mathrm{sp} \text {. } \\
\text { Protozoa }\end{array}$ & 0 & 0 & 72,2 & $1,8(1,9)$ & 26,7 & $0,9(1,9)$ \\
\hline Cryptobia sp. & - & - & 94,0 & - & 80,0 & - \\
\hline Trichodina sp. & - & - & 31,0 & - & 33,0 & - \\
\hline
\end{tabular}




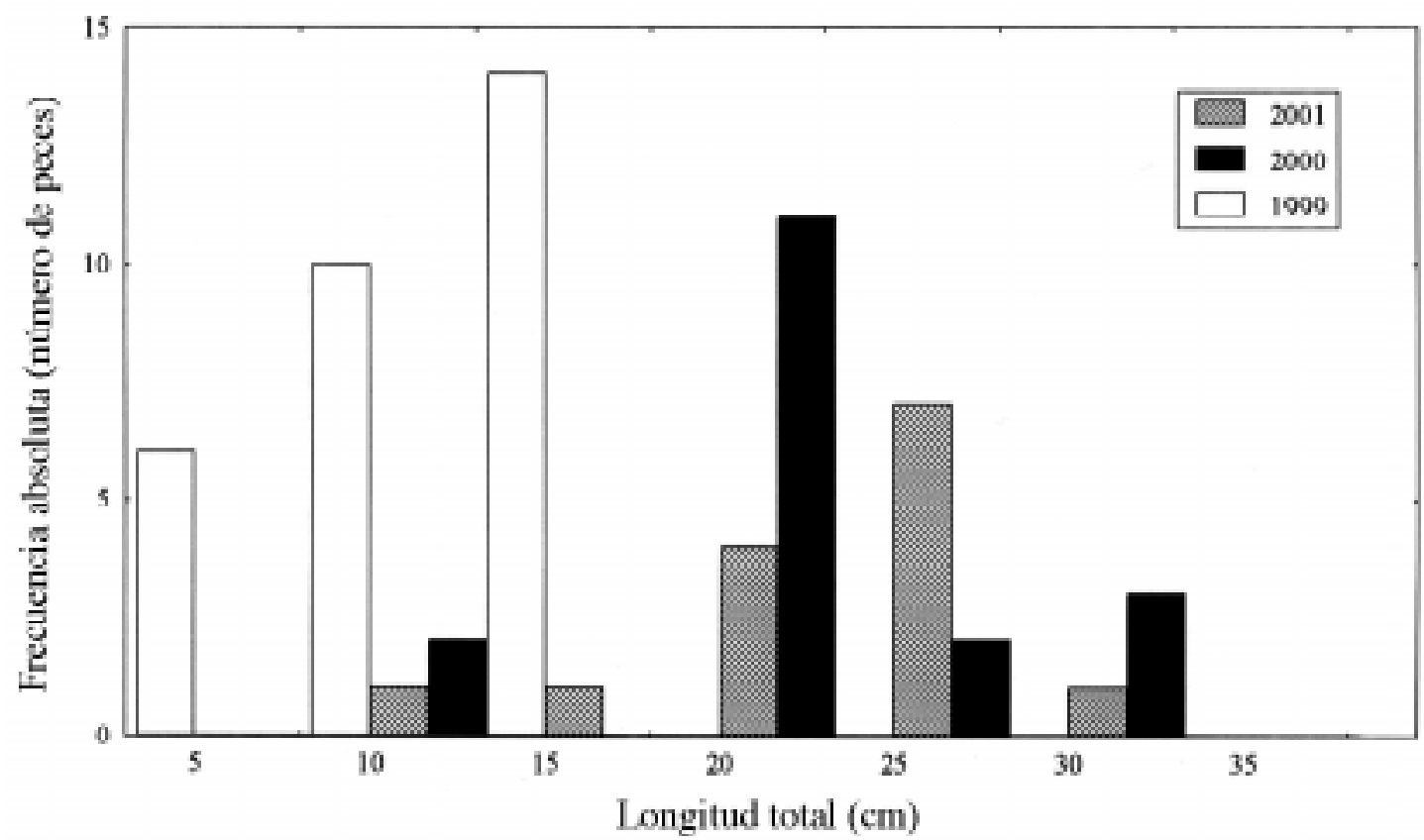

Fig. 1: Distribución de frecuencias absolutas de la longitud total (cm) de 63 ejemplares de Scartichthys viridis muestreados entre los años 1999 y 2001 en la localidad de Las Cruces, Chile central.

Absolute frequency distributions of total body length $(\mathrm{cm})$ of 63 Scartichthys viridis specimens sampled between 1999 and 2001 at Las Cruces, central Chile.

infracomunidades fueran escasos, o comparativamente menores a los cambios cronológicos, y por ende, que en estado adulto las borrachillas sólo tuviesen una mayor abundancia y riqueza de especies en las infracomunidades, por su mayor tamaño corporal, mayor demanda de alimento y mayor tiempo transcurrido desde el nacimiento del huésped.

Se ha recomendado comparar infracomunidades entre huéspedes de similar edad, ya que es usual que haya cambios ontogenéticos en la ecología del huésped que pueden provocar en forma concomitante cambios en ellas (George-Nascimento \& Iriarte 1989, Balboa \& George-Nascimento 1998, Garcías et al. 2001). En términos generales, la variación ontogenética de la dieta del huésped juega un rol importante en el reclutamiento de los endoparásitos ya que puede explicar patrones de abundancia de algunos taxa en función de la longitud corporal del huésped, y por ende, de la composición infracomunitaria. Algo similar se puede esperar de los cambios ontogenéticos en el uso del hábitat. El objetivo de este estudio es averiguar qué tan persistentes son la riqueza, diversidad, abundancia y composición de las infracomunidades, en el tiempo ontogenético y cronológico. Sin embargo, muchas veces es la composición de tamaños de los peces en las mues- tras la que determina qué se puede inferir de estas comunidades.

La borrachilla es eminentemente herbívora, y abundante en ambientes intermareales rocosos (incluyendo pozas intermareales y canales rocosos) a lo largo de la costa de Chile central (Varas \& Ojeda 1990, Muñoz \& Ojeda 2000, Ojeda et al. 2000). Consume macroalgas verdes y rojas, que raspa desde la superficie rocosa con dientes tipo peine (Muñoz \& Ojeda 1997, 2000). Recluta desde el plancton al intermareal rocoso cuando alcanza cerca de $30 \mathrm{~mm}$ de longitud total, y en estado adulto alcanza una longitud total de hasta 300-310 mm (Muñoz \& Ojeda 2000). Este es el primer estudio acerca del parasitismo de esta especie de pez, en que además, se registran los protozoos parásitos, escasamente estudiados en Chile. Se agrega a esto el que el parasitismo de los peces intermareales ha sido escasamente estudiado (Zander et al. 1999).

\section{MATERIALES Y MÉTODOS}

Los ejemplares de $S$. viridis se recolectaron en noviembre de $1999(\mathrm{n}=30)$, diciembre del 2000 $(\mathrm{n}=18)$, y abril del $2001(\mathrm{n}=15)$ desde pozas intermareales vecinas a la Estación Costera de Investigaciones Marinas (ECIM) de la Pontificia 
Universidad Católica de Chile, en Las Cruces, Quinta Región de Chile $\left(33^{\circ} 27^{\prime}\right.$ S, $71^{\circ} 37^{\prime}$ O), Chile central. Los ejemplares se capturaron mediante aplicación del anestésico BZ-20 ${ }^{\circledR}$ al agua de las pozas durante la baja marea. Posteriormente, los ejemplares capturados mediante redes de mano, fueron depositados individualmente en bolsas plásticas y llevados al laboratorio. Allí fueron congelados a $-20^{\circ} \mathrm{C}$, y luego de descongelados, se determinó su longitud total, en $\mathrm{cm}$. En cada individuo huésped se examinó visualmente, a ojo desnudo y bajo lupa o microscopio, la piel, aletas, cavidad bucal y cámara branquial para la búsqueda de ectoparásitos. La masa visceral de cada ejemplar se obtuvo mediante un corte ventral separando el tracto digestivo y las branquias, los cuales fueron disecados y junto a su contenido fueron tamizados bajo la acción de un chorro de agua potable a presión en un cilindro plástico cuyo fondo es una malla de $0,33 \mathrm{~mm}$ de luz. El material retenido en el tamiz fue examinado mediante una lupa para recolectar los parásitos. Los parásitos fueron fijados en formalina al $10 \%$ para su posterior determinación taxonómica, la que se llevó a cabo consultando a Schell (1970) y al Dr. Rodney Bray del Museo de Historia Natural de Londres, Reino Unido para los digeneos, a Whittington \& Horton (1996) para los monogeneos, y a Kabata (1979) para los copépodos. Durante los muestreos de los años 2000 y 2001, y antes de la congelación, se tomaron además, muestras de sangre con capilares desde el seno venoso y muestras de los arcos branquiales para realizar frotis y posteriormente teñirlos por el método Giemsa para buscar protozoos hemoparásitos (Lom \& Dykova 1992, Khan 1998). La abundancia de protozoos no fue evaluada.

Luego de la determinación taxonómica y conteo de los parásitos metazoos, se calculó la prevalencia de cada taxon y del conjunto de ellos, en cada muestra, como el porcentaje de peces infectados. La abundancia total corresponde a la suma de parásitos de cada taxon en cada infracomunidad, independientemente de su identidad taxonómica. La riqueza es el número de taxa parasitarios por infracomunidad. Se supone que se recolectaron todos los parásitos metazoos, por lo que al momento de calcular el índice de diversidad y dominancia de las infracomunidades se utilizó el coeficiente de Brillouin (ver Magurran 1988). El número de huéspedes examinados en cada muestra (entre 15 y 30 peces) se considera razonable ya que las curvas de número de huéspedes examinados y riqueza acumulada de parásitos se estabilizaba entre el octavo y el duodécimo ejemplar.
La persistencia en el tiempo cronológico de la diversidad, riqueza y abundancia total fue evaluada a través de análisis de la varianza de una vía realizados sobre los residuos de la regresión entre dichos descriptores infracomunitarios y la ontogenia del huésped. En dichos análisis el factor era el año de muestreo. En forma específica, en las muestras tomadas en 1999, el primer año de este estudio, se observaron diferencias significativas en la longitud total de los peces respecto de los otros dos años. Este problema fue abordado de dos maneras en el análisis de los datos. Primero, se consideró los datos de los 3 años en conjunto. Segundo, se comparó entre años sólo a muestras de peces de similar longitud total.

La composición de las infracomunidades fue resumida en una dimensión mediante la ordenación de la abundancia relativa de cada taxon en ellas, con un análisis de correspondencia (AC). Luego de la ordenación, se analizaron las variaciones de los puntajes de las infracomunidades en el tiempo cronológico y en el ontogenético en forma similar a los realizados con la diversidad, riqueza, etc. En esta ordenación fueron considerados los ocho taxa de metazoos y las 56 infracomunidades. El único taxon de mínima ocurrencia fue el copépodo Acantochondria sp., que co-ocurría con otros dos taxa, por lo cual no fue necesario eliminarlo de la ordenación (ver Jongman et al. 1995). La distancia entre los puntajes de las infracomunidades en dichas ordenaciones son distancias Chi-cuadrado y están expresadas en unidades de desviación estándar (x 100) de la tasa de reemplazo de los taxa a lo largo del gradiente (Jongman et al. 1995, Legendre \& Legendre 1998). Por esto, la cercanía o lejanía de los taxa en el gradiente representan en parte lo que un índice de similitud y una técnica de clasificación harían en un dendrograma. La bondad de la correspondencia se evalúa con el autovalor (valor propio). Por ello, las especies que están a más de cuatro desviaciones estándar (DE) en el gradiente no co-ocurren en ninguna infracomunidad.

\section{RESULTADOS}

La longitud total de las borrachillas recolectadas en 1999 fluctuó entre 4,8 y 12,9 cm, y resultó ser distinta (prueba Kolmogorov-Smirnov de dos muestras; $\mathrm{P}<0,01)$, y menor que la de los ejemplares de las muestras de los otros dos años, que tuvo un rango entre 10,7 y $33,0 \mathrm{~cm}\left(\mathrm{~F}_{2,60}=94,3\right.$; $\mathrm{P}<0,0001)$. En cambio, la longitud total de los ejemplares recolectados en el año 2000 y en el 2001 no era significativamente diferente (prueba a posteriori de Tukey; 0,60 > P > 0,50; Fig. 1). 
Cincuenta y seis de los 63 huéspedes examinados durante los tres años estaban parasitados con al menos un metazoario $(88,9 \%)$. De ellos se recolectaron 10 taxa parasitarios (dos taxa de protozoos incluidos). De los ocho taxa de metazoos (tres endoparásitos y cinco ectoparásitos) se recolectaron 364 individuos (media $=5,7 ; \mathrm{DE}=5,3$; $\mathrm{n}=63$; Tabla 1). El metazoo de mayor prevalencia y abundancia fue el hirudíneo Oceanobdella sp. Los demás taxa en las muestras ocurrieron en prevalencias y abundancias intermedias y bajas (Tabla 1). Los dos taxa de protozoos encontrados en las borrachillas examinadas en los años 2000 y 2001 eran el flagelado hemoparásito Cryptobia sp., y el ciliado ectoparásito Trichodina sp., que habitaba en la piel y en las branquias. La prevalencia de Cryptobia sp. fue similar y alta en ambos años, y la de Trichodina sp. fue similar entre años, aunque menor que la del hemoflagelado (Tabla 1). La prevalencia total del parasitismo por metazoos no fue significativamente distinta entre pares de años $\left(\chi^{2}\right.$ entre 1,24 y 4,$11 ; 0,95>\mathrm{P}$ $>0,10$; con 1 grado de libertad). Algo similar se observó al comparar la prevalencia de cada protozoario entre años, y según su localización corporal $\left(\chi^{2}\right.$ entre 0,11 y 1,$60 ; 0,98>\mathrm{P}>0,50 ;$ con 1 grado de libertad).

Seis de los ocho taxa de metazoos ocurrieron en los tres años. Uno no estuvo presente en un año (Lepidauchen sp.) y el copépodo Acantochondria sp. fue encontrado solamente en un huésped, y por ende sólo en un año. No hubo diferencias estadísticamente significativas entre años en las prevalencias de infección de los taxa que ocurrieron en los tres años, a excepción de Neobenedenia melleni que fue más prevalente en el año 2001 (Tabla 1).

La correspondencia en el primer eje del AC de los taxa parasitarios y de los huéspedes mostró un valor propio (lambda) de 0,65 y una extensión de 2,53 desviaciones estándar (Fig. 2). Esto indica que hay una correlación de cerca de 0,80 (= raíz de lambda) entre los puntajes de las especies y de los sitios (Pielou 1984), y que la diferencia máxima entre infracomunidades no alcanza a ser total, ya que la extensión del gradiente composicional es menor a cuatro desviaciones estándar (Jongman et al. 1995).

En el conjunto de las muestras, la longitud total de los huéspedes resultó ser un mejor predictor de las variaciones de la riqueza, abundancia, diversidad, dominancia y composición de las infracomunidades de parásitos metazoos, que el año de muestreo (Tabla 2, 3 y 4, Fig. 2 y 3). La longitud total de las borrachillas explica entre el 14 y el $30 \%$ de su varianza (Tabla 3 ). Las variaciones ontogenéticas en la composición de las infracomunidades se observan en el incremento de la abundancia de Paramicrocotyle moyanoi (coeficiente de correlación de Spearman, $\mathrm{r}_{\mathrm{s}}=0,51$; $\mathrm{P}<0,01)$, de Lepeophtheirus sp. $\left(\mathrm{r}_{\mathrm{s}}=0,49 ; \mathrm{P}<\right.$ $0,01)$, y de Lepidauchen sp. $\left(\mathrm{r}_{\mathrm{s}}=0,46 ; \mathrm{P}<0,01\right)$ con la longitud total de las borrachillas. Ningún otro taxon mostró correlaciones significativas en las variaciones de su abundancia con la longitud total del huésped. En cambio, el año de muestreo da cuenta de entre el 5 y el $28 \%$ de la varianza de los descriptores infracomunitarios. De hecho, el efecto significativo del año de muestreo sobre la abundancia, diversidad y riqueza en el conjunto de los datos (Tabla 4) es debida a la muestra de juveniles del año 1999. Así, el año de muestreo no es relevante para explicar las variaciones de la riqueza, abundancia, diversidad, dominancia y composición de las infracomunidades de huéspedes de tamaño similar (de las muestras del 2000 y del 2001, Tabla 4).

\section{DISCUSIÓN}

La ecología poblacional de parásitos busca explicar sus cambios naturales, comprendiendo los factores del medio ambiente, tanto bióticos como

\section{TABLA 2}

Promedio (desviación estándar) de cinco descriptores de las infracomunidades de parásitos y de la longitud total $(\mathrm{cm})$ de las borrachillas muestreadas en Las Cruces, según año de muestreo y para el total; $\mathrm{n}=$ número de observaciones

Arithmetic mean (standard deviation) of five parasite infracommunity descriptors and host body length (cm) of the blennies sampled at Las Cruces, according to year of sampling and the overall sample; $n=$ number of observations

\begin{tabular}{lccccccc}
\hline Año & Abundancia & Riqueza & Diversidad & Dominancia & Composición & Longitud total & $n$ \\
\hline 1999 & $3,83(3,66)$ & $1,47(1,17)$ & $0,37(0,41)$ & $0,58(0,44)$ & $45,6(65,8)$ & $9,4(2,20)$ & 30 \\
2000 & $7,83(6,50)$ & $3,00(1,61)$ & $0,85(0,64)$ & $0,36(0,43)$ & $125,5(61,5)$ & $23,5(5,73)$ & 18 \\
2001 & $7,07(5,31)$ & $2,67(1,54)$ & $0,76(0,57)$ & $0,42(0,40)$ & $141,3(89,8)$ & $24,7(5,18)$ & 15 \\
Total & $5,74(5,26)$ & $2,19(1,54)$ & $0,62(0,57)$ & $0,47(0,43)$ & $95,2(82,5)$ & $17,0(8,50)$ & 63 \\
\hline
\end{tabular}




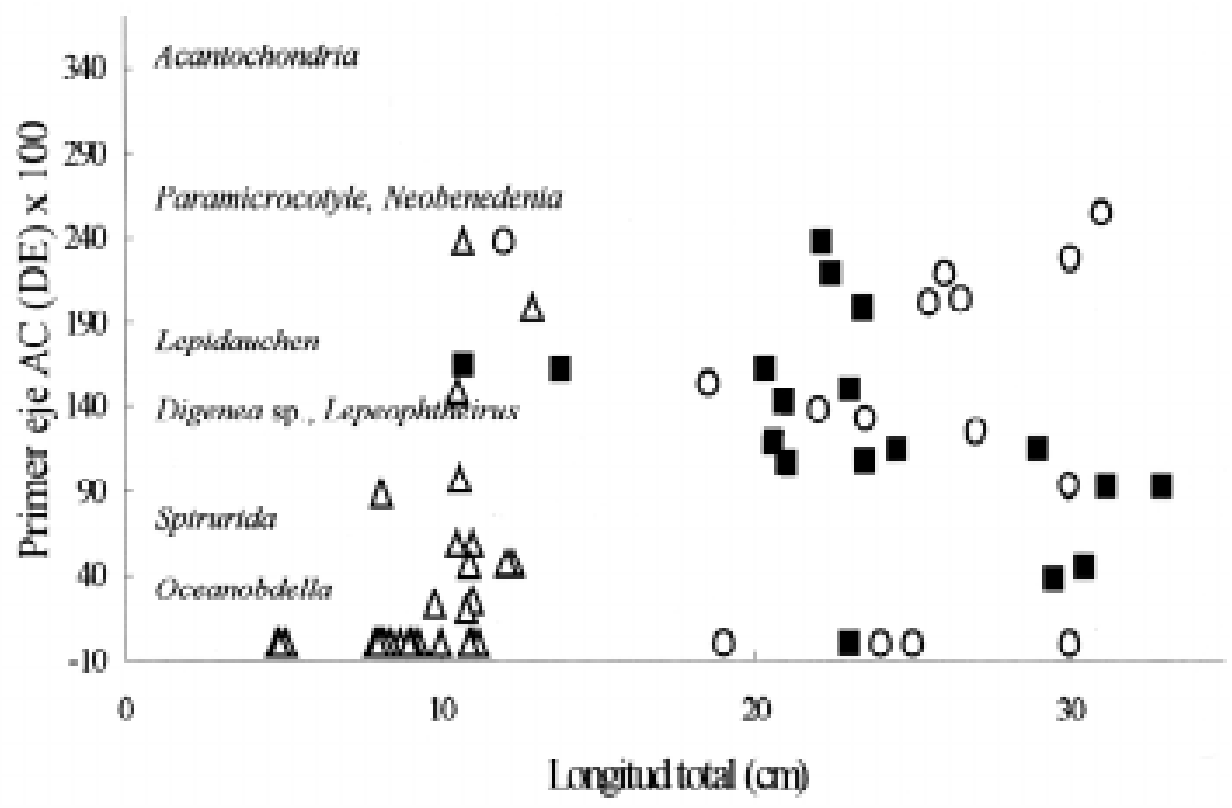

Fig. 2: Relación entre el primer gradiente composicional en el AC de las infracomunidades de parásitos de S. viridis (expresado en unidades de desviación estándar x 100), y la longitud total de los huéspedes (cm), según el año: 30 S. viridis muestreados en 1999 (triángulos), 18 en el 2000 (cuadrados) y 15 en el 2001 (círculos). Los nombres de los taxa parasitarios se indican frente a su posición en el primer eje del AC.

Relationship between the first compositional gradient in AC of the parasite infracommunities in S. viridis (expressed in standard deviation units $\mathrm{x} \mathrm{100)}$, and host body length $(\mathrm{cm})$ according to the year of sampling: $30 \mathrm{~S}$. viridis specimens sampled in 1999 (triangles), 18 in 2000 (squares) and 15 in 2001 (circles). Names of parasite taxa are shown in front of their position on the first AC axis.

abióticos, que afectan el número de ellos en los huéspedes. Las variaciones que experimentan las infracomunidades de parásitos a lo largo de la ontogenia del huésped pueden ser de una medida similar o quizás mayor que la cronológica, en algunas especies de huéspedes, y opuestas en otras. En las comunidades biológicas, este tiempo ontogenético puede ser asimilable al tiempo desde que se "abre" un parche de un determinado tipo de hábitat, luego de una perturbación. En contraste, el tiempo cronológico corresponde al intervalo de meses o años en el que se hacen las observaciones. En el caso de las infracomunidades parasitarias de la borrachilla, la diferencia en compo-

TABLA 3

Coeficientes de determinación $\left(\mathrm{r}^{2}\right)$, en porcentaje, de la relación entre abundancia total, riqueza, diversidad, dominancia y composición de las infracomunidades de parásitos, con la longitud total de $S$. viridis, para cada año y el total de los 3 años de muestreo en Las Cruces. Valores en negrita indican $\mathrm{P}<0,05$. n como en Tablas 1 y 2

Coefficients of determination $\left(\mathrm{r}^{2}\right)$, in percentage, of the relationships between total abundance, richness, diversity, dominance and composition of parasite infracommunities, with total body length of $S$. viridis during each year and for all the 3 years of sampling combined, at Las Cruces. Values in bold are $\mathrm{P}<0,05 . \mathrm{n}$ is the same as in Tables 1 and 2

\begin{tabular}{lccccc}
\hline Año & Abundancia & Riqueza & Diversidad & Dominancia & Composición \\
\hline 1999 & 50 & 41 & 13 & 9 & 16 \\
2000 & 36 & 26 & 25 & 8 & 38 \\
2001 & 5 & 3 & $<1$ & 1 & 7 \\
Total & 32 & 34 & 22 & 7 & 16 \\
\hline
\end{tabular}


sición, abundancia, diversidad y riqueza es menor entre años que entre las edades extremas de una muestra. La persistencia en el tiempo cronológico de los valores de la abundancia, riqueza y diversidad infracomunitaria para huéspedes de similar longitud total (Tabla 4, Fig. 2 y 3 ), hace pensar en algún factor común que determina la complejidad numérica de las infracomunidades. Estos resultados son similares a los encontrados en comunidades de parásitos de otras especies de peces en que hay baja variabilidad infracomunitaria en el tiempo cronológico. Una explicación es que los individuos parásitos persistan largo tiempo en los huéspedes (Holmes 1990, Thoney 1993, Balboa \& George-Nascimento 1998), es decir, una estabilidad producto de la inercia de sistemas con muy bajas tasas de colonización y relativamente altos tiempos de residencia.

El otro tiempo, el ontogenético, tendería a transcurrir en forma repetible en cada infracomunidad. Un aumento de la riqueza y abundancia a través de la ontogenia puede ser el resultado de la acumulación de parásitos de largo promedio de vida, mayor tasa de ingesta en huéspedes de mayor tamaño, e ingesta de presas de mayor tamaño por los huéspedes más grandes (George-Nascimento \& Iriarte 1989). En esta unidad de estudio, dos de los tres taxa parasitarios que mostraron una correlación significativa entre su abundancia y la longitud del huésped, eran ectoparásitos. Por ello, su mayor abundancia puede estar asociada a la mayor superficie corporal del huésped. En cambio, la mayor abundancia del endoparásito Lepidauchen sp. puede deberse a mayores tasas de ingesta "accidental" de pequeños invertebrados asociados a las algas que este pez consume, tales como moluscos, anfípodos y poliquetos. En suma, un aumento de la abundancia, riqueza o diversidad infracomunitaria a través de la ontogenia pudiera ser el resultado de dos mecanismos distintos que operan conjuntamente: el tiempo de vida de los parásitos y las necesidades energéticas del huésped (Balboa \& GeorgeNascimento 1998). Se desconoce la longevidad de cada uno de los taxa encontrados, y respecto a las necesidades energéticas de la borrachilla, se sabe que posee una conducta de forrajeo poco selectiva, que podría atribuirse a las bajas tasas de asimilación que la obligarían al consumo de altas cantidades de alimento (Cáceres \& Ojeda 2000).

Aunque en este estudio se intentó someter a prueba las hipótesis avanzadas por Balboa \& George-Nascimento (1998) y Garcías et al. (2001) en un lapso de 3 años consecutivos, las diferencias en longitud total de los ejemplares muestreados en el primer año condujeron a limitar dicha posibilidad, reduciendo la comparación a sólo los dos últimos años. Con todo, los resultados coinciden con lo previamente registrado para otras especies de huéspedes (Balboa \& GeorgeNascimento 1998, Garcías et al 2001, Muñoz et al. 2001). Esto sugiere que las infracomunidades parasitarias están acotadas no sólo composicionalmente, sino que también numéricamente.

En estudios futuros habría que considerar que los muestreos de largo término pueden entregar información importante en la evaluación de la

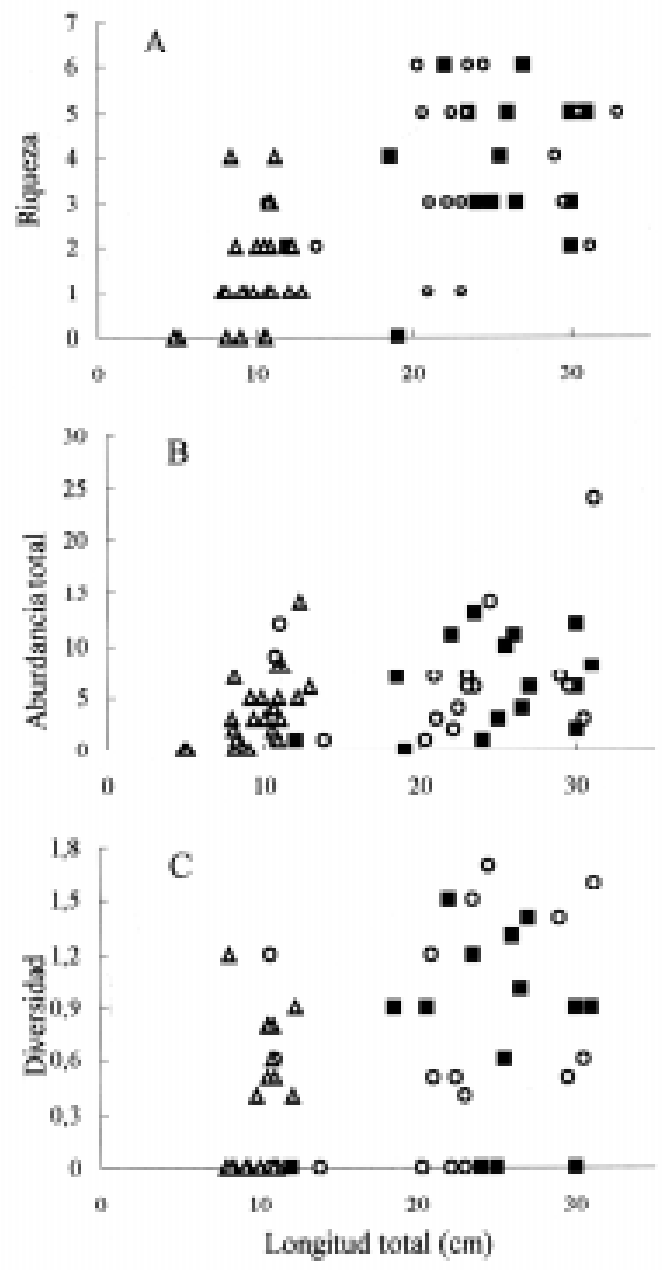

Fig. 3: Relación entre la (A) riqueza, (B) abundancia total, y (C) diversidad de las infracomunidades de parásitos, y la longitud total (cm) del huésped: $30 \mathrm{~S}$. viridis muestreados en 1999 (triángulos), 18 en el 2000 (cuadrados) y 15 en el 2001 (círculos).

Relationship between (A) richness, (B) total abundance, and $(C)$ diversity in parasite infracommunities, and host body length (cm): 30 S. viridis specimens sampled in 1999 (triangles), 18 in 2000 (squares) and 15 in 2001 (circles). 
TABLA 4

Resultados de análisis de la varianza entre años, de la riqueza, abundancia total, diversidad, dominancia y composición de las infracomunidades de parásitos en $S$. viridis (A), y aquellas de longitud media similar (año 2000 y 2001) (B), en Las Cruces. Se indican los valores de F y de probabilidad $(\mathrm{P})$

Results of analyses of the variance between years, of the richness, total abundance, diversity, dominance and composition of parasite infracommunities in S. viridis (A), and those with similar total body length (years 2000 and 2001) (B), at Las Cruces. F-values and probabilities (P) are indicated

\begin{tabular}{lccccc}
\hline \multicolumn{2}{c}{ (A) Todos los datos } & Abundancia & Diversidad & Dominancia & Composición \\
\hline $\mathrm{F}_{(2,60)}$ & 7,94 & 4,28 & $\mathrm{~F}_{(2,53)}=4,85$ & 1,43 & 10,38 \\
$\mathrm{r}^{2}$ & 0,21 & 0,12 & 0,15 & 0,05 & 0,28 \\
$\mathrm{P}$ & $<0,05$ & 0,02 & 0,01 & 0,25 & $<0,001$ \\
\hline (B) Año 2000 y 2001 & \multicolumn{5}{c}{} \\
\hline $\mathrm{F}_{(1,31)}$ & 0,36 & 0,13 & $\mathrm{~F}_{1,30}=0,15$ & 0,18 & 0,35 \\
$\mathrm{P}$ & 0,55 & 0,72 & 0,70 & 0,67 & 0,56 \\
\hline
\end{tabular}

estabilidad temporal de las infracomunidades parasitarias, y que las variaciones estacionales e interanuales que sufren muchas parasitosis de peces podrían modificar los resultados aquí observados. Los principios, patrones y procesos que ayudan a explicar la persistencia temporal de las comunidades biológicas está aún en ciernes. Por ejemplo, la relación entre estabilidad temporal comunitaria, el número de especies presentes y la biomasa y abundancia comunitarias aún se desconoce (Tilman 1999). Este estudio, en suma, señala la necesidad de explorar la estabilidad temporal en más de una dimensión (Garcías et al. 2001).

\section{LITERATURA CITADA}

BALBOA L \& M GEORGE-NASCIMENTO (1998) Variaciones ontogenéticas y entre años de las infracomunidades de parásitos metazoos de dos especies de peces marinos de Chile. Revista Chilena de Historia Natural 71: 27-37.

BEGON M, JL HARPER \& CR TOWNSEND (1990) Ecology: individuals, populations, and communities. Second edition. Blackwell Scientific Publications, Boston, Massachusetts. 929 pp.

CÁCERES C \& FP OJEDA (2000) Patrones de forrajeo en dos especies de peces intermareales herbívoros de las costas de Chile: efecto de la abundancia y composición química del alimento. Revista Chilena de Historia Natural 73: 253-260.

GARCÍAS F, R MENDOZA \& M GEORGE-NASCIMENTO (2001) Variaciones entre años en composición y diversidad de las infracomunidades de parásitos metazoos de la corvina Cilus gilberti (Pisces:Sciaenidae) en Chile. Revista Chilena de Historia Natural 74: 833-840.
GEORGE-NASCIMENTO M \& J IRIARTE (1989) Las infracomunidades de parásitos metazoos del chancharro Helicolenus lengerichi Norman, 1937 (Pisces, Scorpaenidae): un ensamble no interactivo de especies. Revista Chilena de Historia Natural 62: 217-227.

GRUNER L, M MALCZEWSKI, G JAKUB, N BOGUSLAW, K JEDRZEJ \& J BOIUX (1998) Stability of nematode parasite communities of sheep in a Polish flock in relation to years, seasons and resistance status of hosts. Acta Parasitologica 43: 154-161.

HOLMES JC (1990) Helminth communities in marine fishes. En: Esch GW, AO Bush \& JM Aho (eds) Parasite communities: patterns and processes: 101130. Chapman-Hall, New York, New York.

JAKSIC F (2001) Ecología de comunidades. Ediciones Universidad Católica de Chile, Santiago, Chile. 233 pp.

JONGMAN RHG, CJF TER BRAAK \& OFR VAN TONGEREN (1995) Data analysis in community and landscape ecology. Cambridge University Press, New York, New York. 299 pp.

KABATA Z (1979) Parasitic Copepoda of British fishes. Volume 152. The Ray Society, London, United Kingdom. 2017 pp.

KENNEDY CR (1993) The dynamics of intestinal helminth communities in eels Anguilla anguilla in a small stream: long-term changes in richness and structure. Parasitology 107: 71-78.

KENNEDY CR, AO BUSH \& JM AHO (1986) Patterns in helminth communities: why are birds anf fish so different? Parasitology 93: 205-215.

KHAN RA (1998) Parasites of fish as indicators of environmental stress. En: Tada I, S Kojima \& M Tsuji (eds) ICOPA IX International Congress of Parasitology: 313-318. Monduzzi Editore, Bologne, Italy. 
KURIS AM \& KD LAFFERTY (1994) Community structure: larval trematodes in snail hosts. Annual Review of Ecology and Systematics 25: 189-217.

LEGENDRE P \& L LEGENDRE (1998) Numerical ecology. Elsevier Science Publishers, Amsterdam, The Netherlands. 853 pp.

LOM J \& I DYKOVA (1992) Protozoan parasites of fishes. Developments in aquaculture and fisheries science, volume 26. Elsevier Science Publishers, Amsterdam, The Netherlands. 315 pp.

MAGURRAN AE (1988) Ecological diversity and its measurement. Princeton University Press, Princeton, New Jersey. 175 pp.

MUÑOZ AA \& FP OJEDA (1997) Feeding guild structure of a rocky intertidal fish assemblage in central Chile. Environmental Biology of Fishes 49: 471-479

MUÑOZ AA \& FP OJEDA (2000) Ontogenetic changes in the diet of the herbivorous Scartichthys viridis in a rocky intertidal zone in central Chile. Journal of Fish Biology 56: 986-998.

MUÑOZ G, F GARCÍAS, V VALDEBENITO \& M GEORGE-NASCIMENTO (2001) Parasitofauna y alimentación de Notothenia c.f. angustata Hutton, 1875 (Pisces: Nototheniidae) del intermareal de dos localidades del Golfo de Arauco, Chile. Boletín Chileno de Parasitología (Chile) 56: 29-33.

OJEDA FP, FA LABRA \& AA MUÑOZ (2000) Biogeographic patterns of Chilean littoral fishes. Revista Chilena de Historia Natural 73: 625-641.

PIELOU EC (1984) The interpretation of ecological data. John Wiley \& Sons, New York, New York. 263 pp.
PUTMAN RJ (1994) Community ecology. Chapman \& Hall, London, United Kingdom. 178 pp.

SCHELL SC (1970) The trematodes. M.C. Brown Company Publishers, Dubuque, Iowa. 355 pp.

SOUSA WP (1994) Patterns and processes in communities of helminth parasites. Trends in Ecology and Evolution 9: 52-57.

THONEY DA (1993) Community ecology of the parasites of adult spot, Leiostomus xanthurus, and Atlantic croaker, Micropogonias undulatus (Sciaenidae) in the Cape Hatteras region. Journal of Fish Biology 43: 718-804.

TILMAN D (1999) The ecological consequences of changes in biodiversity: a search for general principles. Ecology 80: 1455-1474.

VARAS E \& FP OJEDA (1990) Intertidal fish assemblages of the central Chilean coast: diversity, abundance and trophic patterns. Revista de Biología Marina (Chile) 25: $59-70$.

WHITTINGTON ID \& MA HORTON (1996) A revision of Neobenedenia Yamaguti, 1963 (Monogenea: Capsalidae) including a redescription of $N$. melleni (Mac Callum, 1927) Yamaguti, 1963. Journal of Natural History 30: 1113-1156.

ZANDER CD, LW REIMER \& K BARZ (1999) Parasite communities of the Salzhaff (Northwest Mecklenburg, Baltic Sea). I. Structure and dynamics of communities of littoral fish, especially small-sized fish. Parasitology Research 85: 356-372. 